\title{
Waiting Times in Emergency Department After Using the Emergency Severity Index Triage Tool
}

\author{
Farzad Mahmoodian ${ }^{1}$; Razie Eqtesadi ${ }^{2,3,}$; Atefe Ghareghani ${ }^{4}$ \\ ${ }^{1}$ Department of Medical Ethics, Shiraz University of Medical Sciences, Shiraz, IR Iran \\ ${ }^{2}$ Trauma Research Center, kashan University of Medical Sciences, Kashan, IR Iran \\ 3 Department of Emergency Medicine, Shiraz University of Medical Sciences Shiraz, IR Iran \\ ${ }^{4}$ Shiraz University of Medical Sciences, Shiraz, IR Iran \\ *Corresponding author: Razie Eqtesadi, Trauma Research Center, kashan University of Medical Sciences, Kashan, IR Iran. Tel/Fax: +98-3155620634, E-mail: razieeqtesadi@yahoo.com
}

Received: April 16, 2014; Revised: September 23, 2014; Accepted: November 22, 2014

\begin{abstract}
Background: Hospital emergency departments (EDs) are as barometers of the health care system. Crowded EDs threaten delivery of timely care. Prolonged ED wait times reduce the quality of care and increase adverse and sometimes irreversible events.

Objectives: The purpose of this study was to determine the patients' waiting time at Namazi and Shahid Faghihi hospitals in Shiraz, Iran. Patients and Methods: This analytical cross-sectional study was conducted in two phases from December 2012 to May 2013. First, the researcher attended the EDs of the two hospitals and recorded the information of 900 patients who entered the ED, including arrival time, level of triage, and time of first visit by physician. Then, among patients admitted to the ED units, 273 were followed and waiting times for the first physician order in the referral unit and the commencement of clinical interventions (defined as check time by the nurse) were recorded.

Results: The median waiting time from arrival to first visit by physician for the 900 patients included in the study was 8 (5-14) minutes [median (interquartile range)]. For the patients admitted to referral units, waiting time was 84 (43-145) minutes for the physician order and 85 (45-147) minutes for the commencement of first clinical intervention; 75\% of the patients in triage level I, 84.6\% in triage level II, and 95.6\% in triage level III were visited within the target time limit.

Conclusions: Waiting time for commencement of clinical action in patients admitted to the EDs was considerably high for patients with higher priorities; so, rapid care of critically ill patients, identified during the triage process, should be emphasized.

Keywords:Emergency Department; Efficiency; Triage
\end{abstract}

\section{Background}

Providing timely clinical care is the primary concern of emergency departments (EDs). On the other hand, crowding is a very common problem in EDs, which prolongs the waiting times for patients. Extended waiting time not only increases dissatisfaction with healthcare systems, but also delays admission of new patients and interferes with presenting medical care to the admitted patients (1). Triage systems have been developed to speed up identifying critically ill patients in EDs. Employment of triage scales in EDs has led to significant reduction in waiting times and improved patients satisfaction (2). Chronological studies are the most common methods for examining the efficacy of triage systems (3) and several studies have been conducted in Iran and other countries to evaluate the performance of EDs (4-7). Reducing the waiting times in EDs is an overwhelming problem in health care systems. In the first step for this aim, detailed recording and continuous reporting of EDs performances on waiting times seems essential (8). In Iran, the emergency medicine program has been introduced and developed by national health care systems since
2005 and hospital EDs all over the country are progressively being restructured using international emergency medicine recommendations. The fourth edition of the five-level triage scale, the emergency severity index (ESI), one of the most widely used triage tools, which was last revised in 2004, has been adopted by the National Center for Disaster Management and Medical Emergencies and implemented in hospital EDs nationwide (9). Shiraz is a grand metropolitan area located in southern Iran with traditionally reference medical centers of the region. Emergency medicine specialists have been employed in major hospitals of the city since 2009, but the emergency medicine program has not yet been well-established and ED processes have not been redefined according to the emergency medicine instructions.

\section{Objectives}

The present study was conducted to assess the performance of EDs in two major hospitals of Shiraz city after adoption of ESI version 4 triage scale. Waiting times from entry to the first visit by physician and commencement of diagnostic and therapeutic measures were calculated

Copyright (C) 2014, Kashan University of Medical Sciences. This is an open-access article distributed under the terms of the Creative Commons Attribution-NonCommercial 4.0 International License (http://creativecommons.org/licenses/by-nc/4.0/) which permits copy and redistribute the material just in noncommercial usages, provided the original work is properly cited. 
and the results were compared to similar studies and national target times.

\section{Patients and Methods}

The present cross-sectional study was conducted among patients referring to EDs of Namazi and Shahid Faghihi hospitals in Shiraz, Iran, from December 2012 to May 2013. The two hospitals are among the longstanding hospitals of the city and are the main hospitals located in the city center, traditionally including most of the emergency visits of the metropolis. In both hospitals, the primary assessment is initiated by an experienced triage nurse who records patients' demographic characteristics and vital signs and assigns each patient a priority level from 1 to 5 based on ESI version 4, triage scale. For level 1, medical intervention should begin within one minute, while patients at level 2 can wait up to 10 minutes for medical evaluation and treatment. For levels 3, 4 and 5, the target times for initial evaluation and treatment are defined as 30,60 , and 120 minutes, respectively (9). Therefore, a level 1 patient is directly transmitted to the resuscitation room and medical examination and intervention begins immediately. Patients in higher priority levels get directed to the exam room for visit and examination by physicians. In Namazi Hospital, an emergency medicine specialist and two medical students and in Shahid Faghihi Hospital, an internal medicine resident and two medical students are present in the examination room. First, patients are examined by the medical students; then, if necessary, they report to the specialist or the internal medicine resident and after their approval, the patient gets transferred to the referral ED unit. In the two hospitals, all five levels of patients flow to one place and the patients' flow of levels 4 and 5 ESI triage does not defer from each other. In the referral units, the patients are examined by affiliated residents and further diagnostic or therapeutic measures are prescribed and then checked and performed by nurses. The study was designed in two phases and approved by the Medical Ethics Committee at the Deputy of Research, Shiraz University of Medical Sciences. According to the approval of Medical Ethics Committee, the patients were included in the study using verbal consents. In the first stage, we aimed to determine the patients' waiting times from triage to the first screening visit by physician. After obtaining legal permission for data collection, primary data collection sheets, including case number, age, sex, time of arrival, level of triage and time of first screening visit by physicians were completed; then, the waiting times were calculated as the interval from the time of entry to the ED to the first screening visit by the physician. In the second step, the charts of patients in levels 1, 2, and 3 who were admitted to the referral ED units were investigated by the research assistant. The patients' information including time of arrival, level of triage, time of first prescription order, and time of first clinical measure run and checked by nurses were recorded and waiting times to the first prescription and first clinical intervention (di- agnostic or therapeutic) were also calculated. The whole data gathering and recording process in both hospitals was performed by a research assistant who was a last-year medical student. Neither the patients nor the ED staffs were involved in the data collection process.

The study sample size was calculated based on the total number of patients admitted to both hospitals in the past year as well as the data obtained from the previous studies. The minimum sample size was calculated as 512 and 276 individuals for Namazi and Shahid Faghihi hospitals, respectively. Patients were enrolled in the study using quota sampling method during morning, evening and night shifts, according to the proportions of patients in each shift, based on previous hospital records. Finally, 514 and 386 patients were studied in the first step in Namazi and Shahid Faghihi hospitals, respectively. For the second phase, a minimum sample size of 100 patients was calculated for each center and finally, among 502 patients in levels 1, 2, and 3 hospitalized in the first phase, 273 patient files, including 147 patients in Namazi Hospital and 126 in Shahid Faghihi Hospital were evaluated.

\subsection{Statistical Analysis}

Statistical analysis was performed using SPSS statistical software (version 16). The measures of waiting times calculated in the two hospitals and different levels of triage did not follow a normal distribution; so, the waiting times were reported as medians with interquartile ranges (IQR). Comparisons of different groups were made using nonparametric Mann-Whitney U test and KruskalWallis test. P values less than 0.05 were considered as statistically significant.

\section{Results}

Among 900 patients in the first step, 477 (53\%) were male and 423 (47\%) were female. The mean age of patients was 43 years, $(42.8 \pm 20$ in Namazi Hospital vs. $43.4 \pm 16$ in Shahid Faghihi Hospital, $\mathrm{P}=0.58$ ). The median wait time (IQR) from entry to the first visit by physician was 8 (514) minutes (mean \pm SD: $9.87 \pm 7.55$ ). In Namazi Hospital, the median wait time was 7 (3-11.25) minutes, which was significantly shorter than that of Shahid Faghihi Hospital [10 (6-15) minutes] $(\mathrm{P}<0.001)$. The number of patients in various levels of triage in the two hospitals and the median waiting times in each level are presented in Table 1. Distribution of patients among the triage levels was different in the two hospitals. The proportion of critically ill patients (levels 1 through 3 ) in Namazi Hospital was significantly higher compared with Shahid Faghihi Hospital (59.4\% vs. 51\%; $\mathrm{P}=0.013$ ). To reach a better estimation of performance of the two hospitals from reference to national and international benchmarks, the proportion of patients visited within the triage target times were calculated for each triage level. Overall, $96 \%$ of the patients $(\mathrm{n}=864)$ were examined within the standard timeframe; 95.3\% (490 patients) in Namazi Hospital and 96.9\% (374 
patients) in Shahid Faghihi Hospital; the two hospitals were not significantly different in this regard $(P=0.3)$. In the second phase, among 273 patients in levels 1,2 and 3 admitted to the referral units, the median (IQR) waiting time from entry to the first physician order was 84 minutes (43-145), which was significantly longer in Namazi Hospital [100 minutes (55 -170)] compared with Shahid Faghihi Hospital [66 minutes (36-108)] ( $\mathrm{P}<0.001)$. The median wait time to first clinical intervention was 85 (45147) minutes for the whole admitted patients, 110 (65-190) minutes for Namazi Hospital and 64 (34-106) minutes for Shahid Faghihi Hospital; it was significantly higher at Namazi Hospital compared with Shahid Faghihi Hospi- tal $(\mathrm{P}<0.001)$. Since level-1 patients in Namazi Hospital are transferred to level 2 emergency rooms after primary interventions in the resuscitation room, level-1 and 2 patients were placed in one group for comparisons. The waiting time in different triage levels in the two hospitals and in all of the patients are shown in Table 2 . The median waiting times to first physician order and first clinical intervention were 63 (25-106) and 70 (35-120) minutes for patients transferred by emergency medical services (EMS), and 88 (53-150) and 89 (52-179) minutes for patients with personal admission. Both intervals were significantly shorter for patients received EMS $(\mathrm{P}=0.015$ and $\mathrm{P}=$ 0.002 , respectively).

Table 1. Waiting Times From Entry to the First Visit by Physician in Different Triage Levels ${ }^{a}$, b

\begin{tabular}{|c|c|c|c|c|c|c|}
\hline Level of Triage & Immediate & Emergency & Urgent & Semi-Urgent & Nonurgent & All Patients \\
\hline \multicolumn{7}{|l|}{ Namazi Hospital } \\
\hline Number of patients & $25(4.9)$ & $56(10.9)$ & $224(43.6)$ & $94(18.3)$ & $115(22.4)$ & $514(100)$ \\
\hline Median wait time, IQR & $0(0-2)$ & $4(2-10)$ & $7(4-13)$ & $8(3.75-12.25)$ & $8(5-13)$ & $7(3-11.25)$ \\
\hline Patients visited in target time & $18(72)$ & $47(83.9)$ & $216(96.4)$ & $94(100)$ & $115(100)$ & $490(95.3)$ \\
\hline \multicolumn{7}{|l|}{ Faghihi Hospital } \\
\hline Number of patients & $3(0.8)$ & $80(20.7)$ & $114(29.5)$ & $93(24.1)$ & $96(24.9)$ & $386(100)$ \\
\hline Median wait time, $\mathrm{IQR}^{\mathrm{d}}$ & $0(0-0)$ & $6(3-8)$ & $9(7-14)$ & $12(9-16)$ & $13(9-18)$ & $10(6-15)$ \\
\hline Patients visited in target time & $3(100)$ & $68(85)$ & $114(100)$ & $93(100)$ & $96(100)$ & $374(96.9)$ \\
\hline \multicolumn{7}{|l|}{ All Patients } \\
\hline Number of patients & $28(3.1)$ & $136(15.1)$ & $338(37.6)$ & $187(20.8)$ & $211(23.44)$ & $900(100)$ \\
\hline Median wait time, IQR & $0(0-1.8)$ & $5(3-9)$ & $8(5-13)$ & $10(6-15)$ & $10(6-16)$ & $8(5-14)$ \\
\hline Patients visited in target time & $21(75)$ & $115(84.6)$ & 330 (97.6) & $187(100)$ & $211(100)$ & $864(96)$ \\
\hline
\end{tabular}

a Abbreviation: IQR, interquartile range.

$\mathrm{b}$ Data are presented as No. (\%).

$\mathrm{d}$ The median wait times were significantly different between the two hospitals $(\mathrm{P}<0.05)$.

Table 2. Wait Times From Entry to Triage to the First Physician Order and the First Clinical Measure in Referral Emergency Department Unit ${ }^{\mathrm{a}}$

\begin{tabular}{|c|c|c|c|c|c|c|c|c|c|}
\hline & \multicolumn{3}{|c|}{ Namazi Hospital } & \multicolumn{3}{|c|}{ Faghihi Hospital } & \multicolumn{3}{|c|}{ All Patients } \\
\hline & $\begin{array}{l}\text { Levels } 1 \text { and } \\
2(n=51)\end{array}$ & $\begin{array}{c}\text { Level } 3 \\
(\mathrm{n}=96)\end{array}$ & $\begin{array}{l}\text { All the levels } \\
(\mathrm{n}=147)\end{array}$ & $\begin{array}{c}\text { Levels } 1 \text { and } \\
2(n=88)\end{array}$ & $\begin{array}{l}\text { All the levels } \\
\quad(\mathbf{n}=273)\end{array}$ & $\begin{array}{l}\text { Level 3 } \\
(\mathbf{n}=38)\end{array}$ & $\begin{array}{l}\text { All the levels } \\
\qquad(\mathbf{n}=\mathbf{1 2 6})\end{array}$ & $\begin{array}{l}\text { Levels } 1 \text { and } \\
2(n=139)\end{array}$ & $\begin{array}{c}\text { Level 3 } \\
(\mathrm{n}=134)\end{array}$ \\
\hline $\begin{array}{l}\text { Wait time } \\
\text { until first } \\
\text { physician } \\
\text { order, me- } \\
{\text { dian }(I Q R)^{b}}^{\text {b }}\end{array}$ & $82(30-161)$ & $110(75-175)$ & $100(55-170)$ & $67(33-105)$ & $84(43-145)$ & $64(38-114)$ & $66(36-108)$ & $68(31-121)$ & $95(57-163)$ \\
\hline $\begin{array}{l}\text { Wait time } \\
\text { to first } \\
\text { clinical } \\
\text { measure, } \\
\text { median } \\
(\text { IQR })^{\mathrm{C}}\end{array}$ & $90(39-177)$ & $122(83-191)$ & $110(65-190)$ & $66(32-106)$ & $85(45-147)$ & $58(36-108)$ & $64(34-106)$ & $69(35-125)$ & $105(60-173)$ \\
\hline
\end{tabular}

\footnotetext{
a Abbreviation: IQR, interquartile range

${ }^{b}$ Waiting times to the first physician order were significantly different between the two hospitals $(\mathrm{P}<0.05)$.

${ }^{\mathrm{c}}$ Waiting times to the first clinical measure were significantly different between the two hospitals $(\mathrm{P}<0.05)$.
} 


\section{Discussion}

In the present study, the median waiting times were 8 minutes to first physician visit, 84 minutes to the first referral physician order in ED unit, and 100 minutes to the first clinical action. Although patients waited shorter for the initial screening visit in Namazi Hospital, Shahid Faghihi Hospital had a better performance regarding indices of care delivery in referral ED units. The comparison of waiting times in different triage levels showed that patients with lower levels of triage waited shorter for visit and diagnostic or therapeutic actions. Although this difference was statistically significant, the value of waiting times for patients in higher levels of triage was close to patients with lower levels of triage (the median waiting time for levels 4 and 5 was 10 minutes compared to 0,5 , and 8 minutes for patients in levels 1,2 , and 3, respectively). Although early care of high level patients is helpful, this finding reflected that ED staff did not differentiate considerably between critically ill patients and those with less urgent conditions, which affected the timeliness of care to critically ill patients in the next stages, as the waiting time to first clinical intervention by referral units was considerably longer compared to initial visit. In a similar study conducted by Horwitz and colleagues in 364 non-Federal US hospitals, the mean waiting time for the first medical intervention was 52.4 minutes. The values were $31.8,45.2,58.6$, and 68.6 minutes for triage levels 1, 2, 3 and 4, respectively (10). Waldrop and colleagues reported the mean waiting times of 61,129 , and 182 minutes for patients in levels 1,2 , and 3 respectively (11). Although waiting time for first visit by physician in our study was acceptable compared to recent studies, after the triage, the time of initiation of clinical action in referral units was considerably longer compared to these studies. This difference may arise from problems related to processes after the triage and primary visit, including reception, filing of dossier, lack of beds, hospitalization process, and transfer to the related ED unit. EDs are expected to provide care at least to $90 \%$ of their patients within the triage target times (10). Horwitz and colleagues showed that $78 \%$ of all patients and $66.9 \%$ of critically ill patients in EDs of the studied hospitals were seen by a physician within the triage target and $13.8 \%$ of EDs achieved the triage target for $90 \%$ or more of patients triaged to be seen within an hour (10). In another study, Greene and Hall concluded that $99.3 \%$ of patients at level $1,80.2 \%$ at level $2,69.2 \%$ at level $3,69.7 \%$ at level 4 , and $88.7 \%$ at level 5 were examined within the standard times set for each level (12). In our study, although $96 \%$ of patients were examined in the triage target times, these proportions were $75 \%, 84.6 \%, 97.6 \%, 100 \%$ and $100 \%$ for triage levels 1 to 5, respectively. Poor performance in levels 1, 2, and 3 compared to triage levels 4 and 5 in the studied hospitals and their striking distance from the target times revealed the defective employment of the triage system, especially in the steps following the triage process. The triage process would be efficient, provided that the EDs must prioritize taking care of critically ill patients identified in triage. In our study, $44.2 \%$ of the patients in the two hospitals were in ambulatory priority (triage levels 4 and 5), while in similar studies, the proportion of nonurgent priorities was $12.1 \%$ (10). Rapid management of patients without critical conditions has been proven to be useful to speed up taking care of other triage levels. Cook and colleagues suggest considering a separate unit for handling minor injuries, leading to a significant reduction in accident and emergency departments waiting times (13) and Traverse et al. reduced the waiting times for walkin patients from 35.5 minutes to 19 minutes by placing a senior emergency physician with the triage nurse for examination of nonurgent patients (14). On the other hand, employing a clinical assistant for managing the flow of incoming ED patients have been reported to reduce the waiting time by 4.5 minutes (15). Health care systems are constantly updating their policies and management interventions to improve the EDs performances. ESI version 4 is an efficient and up-to-date tool, which has been validated for various clinical circumstances $(16,17)$. However, implementing an efficient triage system alone is not sufficient to improve the timeliness and quality of emergency services. Rather, more complex interventions are needed including a process redesigning that targets various phases of patient care (18). The long waiting times in the present study resulted from defective implementation of the triage system in stages after assigning the triage levels to patients. To improve the waiting times in the two hospitals, educating the ED staff and careful surveillance of the whole process of patient care are essential and rapid care of critically ill patients identified during the triage process should be endorsed with more emphasis. Restructuring EDs and reorganizing the patient flow through ED processes according to the latest international emergency medicine recommendations to have more efficient ED sections are essential. Besides, due to the high proportion of nonurgent patients referring to these hospitals, adding a rapid assessment clinic for level-4 and 5 patients can considerably reduce the waiting times of level-1, 2, and 3 patients, by excluding the nonurgent patients from EDs' primary responsibility. In our study, we did not investigate the EDs' specified performances in laboratory and imaging sections, we also did not measure the length of stay in ED units. In the second stage, the waiting times were obtained by reviewing the patients' charts for the recorded times, which may not reflect the waiting times precisely; specially, in cases with more critical conditions, the recordings might have been delayed due to the urgency of clinical interventions. This study was a prelude to further and more comprehensive studies to find deficiencies in providing emergency medical care and develop strategies to overcome barriers and exert necessary changes at different stages of providing medical care in hospital EDs, with the aim of constant improvement of the quality of care. 


\section{Acknowledgements}

The authors would like to gratefully thank the triage personnel of Namazi and Faghihi hospitals for their support.

\section{Authors' Contributions}

Razie Eqtesadi: study design and article writing; Farzad Mahmoodian: study design; Atefe Ghareghani: data collection.

\section{References}

1. Sprivulis PC, Da Silva JA, Jacobs IG, Frazer AR, Jelinek GA. The association between hospital overcrowding and mortality among patients admitted via Western Australian emergency departments. Med JAust. 2006;184(5):208-12.

2. Taylor C, Benger JR. Patient satisfaction in emergency medicine. Emerg Med J. 2004;21(5):528-32.

3. Institute of Medicine Committee on the Future of Emergency Care in the US Health System.. Hospital-based emergency care: at the breaking point. Natl Acad Sci. 2006.

4. Yamani N, Moosavi SA, Alizadeh M, Khorvash F, Ghiasi M.A360-degree performance evaluation of emergency medicine ward in Alzahra Hospital. J Pak Med Assoc. 2012;62(3 Suppl 2):S13-7.

5. Ajami S, Ketabi S, Yarmohammadian MH, Bagherian H. Wait time in emergency department (ED) processes. Med Arh. 2012;66(1):53-

6. Lambe S, Washington DL, Fink A, Laouri M, Liu H, Scura Fosse J, et al. Waiting times in California's emergency departments. Ann Emerg Med. 2003;41(1):35-44.

7. Banerjea K, Carter AO. Waiting and interaction times for patients in a developing country accident and emergency department. Emerg Med J. 2006;23(4):286-90.
8. Wilper AP, Woolhandler S, Lasser KE, McCormick D, Cutrona SL, Bor DH, et al. Waits to see an emergency department physician: U.S. trends and predictors, 1997-2004. Health Aff(Millwood). 2008;27(2):w84-95.

9. Gilboy N, Tanabe P, Travers D. Emergency Severity Index, Version 4 Implementation Handbook.Rockville: Agency for Healthcare Research and Quality; 2005.

10. Horwitz LI, Green J, Bradley EH. US emergency department performance on wait time and length of visit. Ann Emerg Med. 2010;55(2):133-41.

11. Waldrop RD, Harper DE, Mandry C. Prospective assessment of triage in an urban emergency department. South Med J. 1997;90(12):1208-12.

12. Greene J, Hall J. The comparability of emergency department waiting time performance data. Med J Aust. 2012;197(6):345-8

13. Cooke MW, Wilson S, Pearson S. The effect of a separate stream for minor injuries on accident and emergency department waiting times. Emerg Med J. 2002;19(1):28-30.

14. Travers JP, Lee FC. Avoiding prolonged waiting time during busy periods in the emergency department: Is there a role for the senior emergency physician in triage? Eur J Emerg Med. 2006;13(6):342-8.

15. Huang EP, Liu SS, Fang CC, Chou HC, Wang CH, Yen ZS, et al. The impact of adding clinical assistants on patient waiting time in a crowded emergency department. Emerg Med J. 2013;30(12):1017-9.

16. Elshove-Bolk J, Mencl F, van Rijswijck BT, Simons MP, van Vugt AB. Validation of the Emergency Severity Index (ESI) in self-referred patients in a European emergency department. Emerg Med J. 2007;24(3):170-4.

17. Green NA, Durani Y, Brecher D, DePiero A, Loiselle J, Attia M Emergency Severity Index version 4: a valid and reliable tool in pediatric emergency department triage. Pediatr Emerg Care. 2012;28(8):753-7.

18. Storm-Versloot MN, Vermeulen H, van Lammeren N, Luitse JS, Goslings JC. Influence of the Manchester triage system on waiting time, treatment time, length of stay and patient satisfaction; a before and after study. Emerg Med J. 2014;31(1):13-8. 